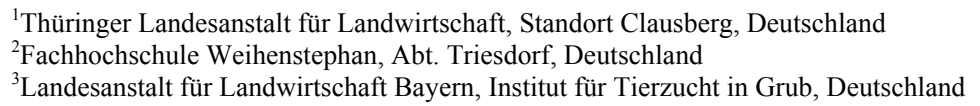

\title{
Futteraufnahme und Fressverhalten wachsender Lämmer 2. Mitteilung: genetische Korrelationen zwischen der Futteraufnahme und Leistungsmerkmalen
}

\begin{abstract}
Title of the paper: Feed intake and eating behaviour of growing lambs. $2^{\text {nd }}$ communication: genetic correlations between feed intake and performance traits

In the present study genetic correlations between feed intake, feed efficiency and daily feed intake on one hand and fattening performance, carcass quality and fertility on the other hand were estimated. Data were collected in the test stations "Schöndorf"/Thuringia and in "Grub"/Bavaria as well as in stud book farms in Thuringia and Bavaria. Fattening performance and carcass quality were measured on 4,397 and 2,264 lambs, respectively, in Schöndorf and 1,292 lambs in Grub.

The genetic correlation between feed efficiency and the number of lambs born alive (LGL) was in Thuringia $r_{A}=-0.35$, but in Bavaria -0.03 only. Further, the genetic correlations between daily feed intake and LGL were different with 0.29 in Thuringia and -0.20 in Bavaria. But the standard errors of the estimates were high due to an insufficient genetic linkage between data from station testing (fattening performance, carcass quality) and from field testing (fertility). Therefore, the results have to be considered as tendencies. A high feed intake during the whole test period led to better daily gain. The genetic relationships between feed intake and carcass quality were indifferent. When using feed intake or efficiency for breeding value estimation and selection no basically problems have to be expected.
\end{abstract}

Keywords: sheep breeding, feed intake, feed efficiency, genetic correlations, fertility fattening performance and carcass quality

\section{Zusammenfassung}

In der vorliegenden Mitteilung stehen die genetischen Korrelationen zwischen der Futteraufnahme, der Mastleistung, dem Schlachtkörperwert und der Fruchtbarkeit im Vordergrund. Für die Analyse standen Daten aus den Prüfstationen Schöndorf/Thüringen und Grub/Bayern sowie aus Herdbuchbetrieben beider Bundesländer zur Verfügung. Die Mastleistung und der Schlachtkörperwert wurden an 4.397 bzw. 2.265 Lämmern in Schöndorf und an 1.292 Lämmern in Grub erfasst.

Zwischen dem Futteraufwand und der Anzahl lebend geborener Lämmer (LGL) betrug die Korrelation in Thüringen $\mathrm{rA}=-0,35$, in Bayern dagegen nur -0,03. Auch die genetischen Korrelationen der täglichen Futteraufnahme zur LGL zeigten sich widersprüchlich (Thüringen +0,29; Bayern -0,20).

Allerdings waren die entsprechenden Standardfehler aufgrund ungenügender genetischer Verknüpfungen zwischen den Stationsdaten (Mastleistung, Schlachtkörperwert) und den Felddaten (Fruchtbarkeit) hoch, so dass die Aussagesicherheit eingeschränkt ist. Eine hohe Futteraufnahme führte zu höheren Zunahmen. Hinsichtlich der Schlachtkörperwerte waren die genetischen Beziehungen uneinheitlich. Grundsätzliche Probleme bei Einbeziehung der Futterdaten in Zuchtwertschätzung und Selektion sind nicht zu erwarten.

Schlüsselwörter: Schafzucht, Futteraufnahme, Futterverwertung, genetische Korrelationen, Fruchtbarkeit, Mastund Schlachtleistung 


\section{Einleitung}

Während die Mutterschafe überwiegend marginale Grünlandflächen nutzen, absolvieren ihre Lämmer oft eine intensive Mast mit hohem Kraftfutteranteil in der Ration. Hierbei sollten die Mutterschafe eine hohe Fruchtbarkeit und die Lämmer hohe Zunahmen erreichen. Somit müssen bei der Berücksichtigung der Futteraufnahme in der Zuchtarbeit ihre Beziehungen zu den wichtigsten Leistungsmerkmalen untersucht werden.

In der vorliegenden Untersuchung bestand das Ziel in der Schätzung genetischer Korrelationen zwischen Merkmalen der Futteraufnahme einerseits, sowie Merkmalen der Fruchtbarkeit, der Mastleistung und des Schlachtkörperwertes andererseits.

\section{Material und Methoden}

\section{Datenmaterial und Tiere}

\section{Mast- und Schlachtleistung}

Die Daten der Mastleistung und des Schlachtkörperwertes wurden in den Prüfstationen Grub (Bayern) und Schöndorf (Thüringen) erfasst. Aus Grub gelangten Daten von 1999 bis 2006 und aus Schöndorf von 1996 bis 2006 in die Untersuchung. Die Futteraufnahme wird in Schöndorf seit 2003 erfasst. Neben Merinolandschafen absolvierten Merinolangwollschafe die Prüfung in Schöndorf, während in Grub ausschließlich Merinolandschafe aufgestallt wurden.

In den Prüfstationen Schöndorf und Grub wurden die abgesetzten Lämmer im Gewichtsbereich zwischen 20 und $42 \mathrm{~kg}$ getestet. In Schöndorf gelangte ein Teil der Prüflämmer in die Zucht und der andere Teil mit gemerzten Tieren wurde in kommerziellen Schlachthöfen geschlachtet. Hierbei steht die Nutzung des Ultraschalls am lebenden Tier im Vordergrund (GERNAND und LENZ, 2005). An die Gruber Station ist ein Schlachthaus angegliedert, in dem alle geprüften Lämmer geschlachtet und zerlegt wurden.

Folgende Daten standen zur Beschreibung der Mastleistung und des Schlachtkörperwertes zur Verfügung:

- beide Stationen

LTZ (g/Tag) Lebenstagszunahme in Gramm pro Tag von der Geburt bis zum Ende der Prüfung

PTZ (g/Tag) Prüftagszunahme in Gramm pro Tag vom Beginn bis zum Ende der Prüfung

SKK (1-9) Beurteilung des Schlachtkörpers - Keule

SKR (1-9) Beurteilung des Schlachtkörpers - Rücken

SKB (1-9) Beurteilung des Schlachtkörpers - Blatt bzw. Schulter

SKF (1-9) Beurteilung des Schlachtkörpers - Fettauflage (1 sehr fett bis 9 sehr mager)

NF (g) Nierenfettmenge in Gramm

SKG $(\mathrm{kg}) \quad$ Schlachtkörpergewicht

- Schöndorf

USM (kg) Lebendgewicht am Tag der Ultraschallmessung gegen Ende der Prüfung

USK (mm) Muskeldicke (Musculus longissimus dorsi) mittels Ultraschallmessung auf Höhe der letzten Rippe 
USF (mm) Rückenfettdicke mittels Ultraschallmessung auf Höhe der letzten Rippe

- Grub
$\mathrm{KBR}(\mathrm{cm})$
Breite des Musculus longissimus dorsi
KFL $\left(\mathrm{cm}^{2}\right) \quad$ Fläche des Musculus longissimus dorsi.

Einen Überblick über die statistischen Maßzahlen der Daten vermittelt Tabelle 1.

Tabelle 1

Anzahl Tiere (n), Rohmittelwert ( $\overline{\mathrm{x}}$ ), Standardabweichung (S), Minimum (Min), Maximum (Max) für Merkmale der Mastleistung und des Schlachtkörperwertes in Schöndorf und Grub

(Number of tested lambs [n], mean [ $\overline{\mathrm{x}}$ ], phenotypic standard deviation [S], minimum [Min], maximum [Max] for fattening performance and carcass quality in "Schöndorf" and "Grub")

\begin{tabular}{|c|c|c|c|c|c|c|}
\hline & $\mathrm{N}$ & Min & Max & $\overline{\mathrm{x}}$ & $\mathrm{S}$ & VK $(\%)$ \\
\hline \multicolumn{7}{|c|}{ Schöndorf } \\
\hline LTZ & 4.396 & 151 & 740 & 340,6 & 64,8 & 19,1 \\
\hline PTZ & 4.397 & 195 & 763 & 408,8 & 56,9 & 13,9 \\
\hline SKK & 2.265 & 3 & 9 & 6,7 & 0,8 & 11,9 \\
\hline SKR & 2.264 & 3 & 9 & 6,5 & 1,0 & 15,4 \\
\hline SKB & 2.264 & 2 & 9 & 6,5 & 0,8 & 12,3 \\
\hline SKF & 2.263 & 4 & 9 & 6,4 & 0,7 & 10,9 \\
\hline NF & 2.228 & 29 & 999 & 199,3 & 84,6 & 42,4 \\
\hline USK & 4.058 & 31 & 400 & 264,6 & 27,8 & 10,5 \\
\hline USF & 4.047 & 3 & 70 & 21,7 & 7,6 & 35,0 \\
\hline \multicolumn{7}{|l|}{ Grub } \\
\hline LTZ & 1.236 & 155 & 533 & 318,4 & 59,4 & 18,7 \\
\hline PTZ & 1.236 & 265 & 617 & 421,9 & 52,1 & 12,3 \\
\hline SKK & 1.289 & 6 & 9 & 7,3 & 0,6 & 8,2 \\
\hline SKR & 823 & 5 & 9 & 7,2 & 0,6 & 8,3 \\
\hline SKB & 1.289 & 4 & 9 & 7,0 & 0,8 & 11,4 \\
\hline SKF & 1.289 & 5 & 9 & 7,5 & 0,6 & 8,0 \\
\hline NF & 1.292 & 60 & 525 & 245,5 & 71,4 & 29,1 \\
\hline KBR & 1.289 & 19 & 23 & 21,0 & 0,6 & 2,9 \\
\hline KFL & 1.284 & 10 & 20 & 14,4 & 1,7 & 11,8 \\
\hline
\end{tabular}

\section{Fruchtbarkeit}

Für die Untersuchung standen die Herdbuchdaten der Merinoland- (ML) und Merinolangwollschafe (MLW) des Landesverbandes Thüringer Schafzüchter der Jahre 1996 bis 2006 zur Verfügung. Aus Bayern wurden alle im Herdbuch erfassten Ablammungen der Merinolandschafe von 1995 bis 2006 zur Verfügung gestellt.

Insgesamt waren aus der Thüringer Herkunft Daten von 52.692 Schafen (ML: 8.745; MLW: 43.947) und von 16.515 Lammungen (MLW: 13.753; ML: 2.762) auswertbar. Aus Bayern standen die Daten von 40.126 Lammungen von 9.827 Schafen zur Verfügung. Da Zulassungstermine und Bedeckungen nicht immer konkret erfasst wurden, konnte nur die Anzahl lebend geborener Lämmer (LGL) der erfassten Lammungen ausgewertet werden. Lammungen ohne lebendgeborenes Lamm wurden nicht einbezogen, da die überwiegende Mehrzahl dieser Lammungen zu keinem Eintrag in das Herdbuch führte.

Die wenigen höhergradigen Mehrlinge (Drillinge und mehr) (Tabelle 2) wurden gemeinsam mit den Zwillingen zu Mehrlingen zusammengefasst. Damit ergab sich die Möglichkeit, die Anzahl Lämmer als bivariates Merkmal zu behandeln. 
Tabelle 2

Häufigkeiten von Einlings- und Mehrlingsgeburten für lebend geborene Lämmer in Thüringen und Bayern (Frequency of single and multiple births for lambs born alive in Thuringia and Bavaria)

\begin{tabular}{lcccc}
\hline Lämmer/Lammung & 1 & 2 & Anzahl Lämmer & \\
& Thüringen & & & Summe \\
$\mathrm{N}$ & 28.132 & 23.680 & 880 & \\
$\%$ & 53,4 & 44,9 & 1,7 & 52.692 \\
Bayern & & & & 100 \\
$\mathrm{~N}$ & 15.861 & 22.595 & 1.670 & 40.126 \\
$\%$ & 39,5 & 56,3 & 4,2 & 100 \\
\hline
\end{tabular}

\section{Genetische Verknüpfung}

$\mathrm{Da}$ in der Mastleistungsprüfung nur männliche Lämmer geprüft wurden, gab es kein Tier, zu dem Leistungsinformationen sowohl aus der Stationsprüfung als auch zur Fruchtbarkeit vorlagen. Allerdings hatten 170 Thüringer Böcke mehr als einen und 137 Thüringer Böcke mehr als 5 Nachkommen in beiden Leistungsbereichen.

Bezüglich der Futterdaten und der Fruchtbarkeit waren es 40 Thüringer Böcke mit mehr als einem Nachkommen und 26 Böcke mit mehr als 5 Nachkommen in beiden Leistungsbereichen. In Grub waren es insgesamt 61 Böcke mit mehr als zwei und 32 Böcke mit mehr als fünf Nachkommen in beiden Leistungskomplexen.

Die auf dieser Basis zu schätzenden genetische Korrelationen zwischen den Merkmalskomplexen müssen relativ unsicher bleiben, wesentliche genetische Beziehungen zwischen den Merkmalen können aber erkannt werden.

\section{Methoden}

Die Varianzkomponenten wurden mit dem Programmpaket VCE, Version 5.1 (KOVAC et al., 2003) bei Einsatz des analytischen Gradienten geschätzt.

Für die genetischen Korrelationen kamen multivariate Schätzläufe zum Einsatz, wobei die jeweiligen Blöcke an Merkmalen gegenübergestellt wurden. Führten diese Schätzläufe nicht zu Resultaten, die eine Schätzung von Standardfehlern zulassen, wurden sie durch bivariate Schätzungen ersetzt.

\section{Modellwahl}

Die Modelle für die Fruchtbarkeitsdaten sind bei GERNAND et al. (2007) beschrieben.

Für die Merkmale des Schlachtkörperwertes kamen Modelle, die denen der RoutineZuchtwertschätzung aus Thüringen entsprechen, zur Anwendung. Sie sind bei GERNAND und LENZ (2005) beschrieben. Diese Modelle wurden auf die bayerischen Daten übertragen. Tabelle 3 enthält die Einflussfaktoren der untersuchten Merkmale.

\section{Ergebnisse und Diskussion}

\section{Beziehung zwischen der Futteraufnahme und der Fruchtbarkeit}

Die genetischen Beziehungen zwischen der Futteraufnahme und dem Futteraufwand einerseits sowie der Fruchtbarkeit andererseits sind aufgrund der hohen Standardfehler nicht gesichert (Tabelle 4). Hierbei ist zu berücksichtigen, dass nur wenige Böcke vorhanden waren, deren Nachkommen einerseits stationär und andererseits im Feld 
geprüft wurden. Eine Erweiterung der Datengrundlage ist anzustreben, um mit einer intensiveren genetischen Verknüpfung zwischen der Futteraufnahme und der Fruchtbarkeit genetische Parameter zu schätzen.

Tabelle 3

Im Modell berücksichtigte Einflussfaktoren für die Merkmale der Mastleistung und des Schlachtkörperwertes (In the statistical model included effects for traits of fattening performance and carcass quality)

\begin{tabular}{|c|c|c|c|c|c|c|c|c|c|}
\hline Merkmal & $\begin{array}{l}\text { Einstallungs- } \\
\text { quartal }\end{array}$ & $\begin{array}{l}\text { Schlacht- } \\
\text { datum }\end{array}$ & $\begin{array}{l}\text { Ultraschall- } \\
\text { datum }\end{array}$ & Rasse & Geburtstyp & $\begin{array}{l}\text { Herkunfts- } \\
\text { betrieb }\end{array}$ & $\begin{array}{c}\text { Schlacht- } \\
\text { körpermasse* }\end{array}$ & $\begin{array}{l}\text { Körpermasse } \\
\text { Ultraschall* }\end{array}$ & $\begin{array}{c}\text { Herde, Jahr, } \\
\text { Saison im } \\
\text { Feld } \\
\end{array}$ \\
\hline$\overline{\mathrm{ZA}}$ & & & & + & + & + & & & + \\
\hline PTZ & + & & & + & & + & & & \\
\hline SKK & & + & & + & & + & + & & \\
\hline SKR & & + & & + & & + & + & & \\
\hline SKB & & + & & + & & + & + & & \\
\hline SKF & & + & & + & & + & + & & \\
\hline NF & & + & & + & & + & + & & \\
\hline USK & & & + & + & & + & & + & \\
\hline USF & & & + & + & & + & & + & \\
\hline KBR & & + & & + & & + & + & & \\
\hline KFL & & + & & + & & + & + & & \\
\hline
\end{tabular}

* Covariable

Dennoch deuten die Ergebnisse aus Thüringen an, dass Zuchtböcke, deren Nachkommen in der Prüfstation das Futter effizient verwerteten, fruchtbarere Töchter hervorbrachten. Dies ist an der Schöndorfer Korrelation von $\mathrm{r}_{\mathrm{A}}=-0,35$ zwischen FAW und LGL abzulesen. Weiterhin waren die Töchter von Zuchtböcken, deren stationsgeprüfte Nachkommen ein großes Futteraufnahmevermögen pro Tag aufwiesen, fruchtbarer. Allerdings zeigen die Ergebnisse aus Bayern diese Tendenz nicht, sodass noch keine endgültigen Schlussfolgerungen zu ziehen sind. Die Ursachen für die unterschiedlichen Ergebnisse könnten in den verschiedenen Versorgungssituationen der Mutterschafe liegen. So werden in Bayern $22 \%$ der Trächtigkeiten aus Bedeckungen zwischen März und Juni erzielt, in Thüringen nur $8 \%$. Bedeckungen im August und September, den Monaten mit dem infolge Sommertrockenheit vermutlich schlechtesten Weidefutter, machten in Thüringen $48 \%$ in Bayern nur $31 \%$ aus. Diese und andere Abweichungen der Bedingungen für die Mutterschafe waren eventuell für die unterschiedlichen Ergebnisse verantwortlich. Möglicherweise hatte eine Variation der aufgenommenen Futtermenge nur in Mangelsituationen, die in Thüringen wahrscheinlich häufiger waren, Bedeutung für die Fruchtbarkeit.

Aufgrund der in Thüringen beobachteten Tendenzen ist eine Fortführung der Untersuchungen an einem größeren Datensatz erforderlich.

Tabelle 4

Genetische Korrelationen (Standardfehler als Subskript) zwischen der Futteraufnahme (FAP), dem Futteraufwand (FAW) und der täglichen Futteraufnahme (TFM) einerseits und der Fruchtbarkeit (LGL) andererseits in Schöndorf und Grub

(Genetic correlations [standard error as subscript] between feed intake [FAP], feed efficiency [FAW], daily feed intake [TFM] on one hand and fertility [LGL] on the other hand in "Schöndorf" and "Grub")

\begin{tabular}{lcccccc}
\hline Merkmal & FAP & $\begin{array}{c}\text { Schöndorf } \\
\text { FAW }\end{array}$ & TFM & FAP & FAW & TFM \\
\hline LGL & $-0,12_{0,26}$ & $-0,35_{0,23}$ & $0,29_{0,16}$ & $-0,02_{0,10}$ & $-0,03_{0,10}$ & $-0,20_{0,02}$ \\
\hline
\end{tabular}


Beziehung zwischen der Futteraufnahme einerseits und der Mastleistung sowie dem Schlachtkörperwert andererseits

Die genetischen Korrelationen zwischen der Futteraufnahme und dem Futteraufwand einerseits und den Merkmalen der Mastleistung und des Schlachtkörperwertes andererseits sind der Tabelle $5 \mathrm{zu}$ entnehmen. Da für die multivariaten Schätzläufe keine Standardfehler $\mathrm{zu}$ ermitteln waren, sind die Ergebnisse der bivariaten Rechenläufe aufgeführt.

Die genetischen Korrelationen waren zwischen der Futteraufnahme und dem Futteraufwand einerseits und der Prüftagszunahme stark negativ.

Geprägt sind diese Beziehungen vom niedrigeren Erhaltungsbedarf, wenn die Mastphase schneller durchlaufen wird. Grundsätzlich andere Ergebnisse müssen sich bei zeitabhängiger Prüfung für die Futteraufnahme ergeben. SNOWDER und VAN VLECK (2005) ermittelten stark positive genetische Korrelationen $(0,99)$ zwischen Futteraufnahme und Zunahmeleistung. Untersuchungen an Fleischrindern zeigten, dass mit zunehmender Zuwachsleistung die Futtereffizienz steigt (MAC NEIL, 1991; FAN et al., 1995; ARCHER et al., 1997, CASTRO BULLE et al., 2007). KORMAN (2001) schlägt zur Verbesserung der Effizienz vor, bei limitierter Fütterung auf Zunahme zu selektieren.

Auch die Zunahme in der Aufzuchtphase vor der Prüfung korreliert deutlich in die gleiche Richtung, ohne dass dies durch die eher niedrige genetische Korrelation der Zunahmeleistungen in den verschiedenen Phasen (GERNAND und LENZ, 2005) erklärt werden könnte.

Wird dagegen die tägliche Futteraufnahme betrachtet, so wird deutlich, dass gut fressende Lämmer wie erwartet auch eine höhere Zuwachsleistung zeigen.

$\mathrm{Zu}$ ähnlichen Ergebnissen gelangten CAMMACK et al. (2005). Sie schätzten eine Korrelation von $\mathrm{r}_{\mathrm{A}}=0,80$ zwischen der täglich aufgenommenen Futtermenge und der Prüftagszunahme.

Liegt die Zunahmeleistung in der Aufzuchtphase vor der Prüfung dagegen hoch, erreichen die Tiere sehr jung das Startgewicht zur Prüfung. Möglicherweise liegt hier die Ursache zwischen der negativen genetischen Korrelation zur täglichen Futteraufnahme in der Prüfphase.

Die Korrelationen zwischen der Futteraufnahme und dem Futteraufwand einerseits und den Schlachtkörpermerkmalen andererseits waren aufgrund unterschiedlicher Vorzeichen nur schwer zu interpretieren. In Thüringen zeigte die Rückenbeurteilung deutlich positive Korrelationen zu Futteraufnahme und Futteraufwand, während die Ultraschallwerte das Gegenteil dokumentierten.

Die Keule scheint dagegen eher zu sehr niedrigen oder negativen Korrelationen zu den kumulierten Futterwerten zu tendieren. In Schöndorf traf dies auf die Beurteilungsnoten zu, während in Grub die Keulenbreite betroffen war. Auch für die Fettmerkmale zeigte sich keine einheitliche Tendenz. Im Schöndorfer Material wies nur die Schlachtkörperbeurteilung nicht aber die Nierenfettmenge oder der Ultraschallwert eine erkennbare genetische Beziehung zu den kumulierten Futtermerkmalen auf. In Grub dagegen war auch die Korrelation zwischen den Futtermerkmalen und der Nierenfettmenge deutlich größer als Null.

Dass eine schlechtere Futtereffizienz in einer genetischen Beziehung zu fetteren Schlachtkörpern stehen könnte, war aufgrund der niedrigen Tierzahl von NKRUMAH et al. (2007) an Fleischrindern nur anzudeuten. 
Die täglich aufgenommene Futtermenge (TFM) lässt den Schluss zu, dass in Schöndorf Tiere mit fetteren Schlachtkörpern mehr fressen. Insbesondere die Beziehung zum Ultraschallwert für Fettauflage von $r_{A}=0,59$ macht dies deutlich, während die anderen Fettwerte nur Korrelationen um 0,3 zeigen. In Grub dagegen blieben alle genetischen Korrelationskoeffizienten der Schlachtkörpermerkmale zur täglich aufgenommenen Futtermenge niedrig.

Auch die bisher vorliegenden Untersuchungen an Fleischrindern lassen keine einheitliche Tendenz erkennen.

Phänotypisch konnten BAKER et al. (2006) im Gegensatz zu CASTRO BULLE et al. (2007) die erwartete Beziehung zwischen Residualfuttermenge und Schlachtkörperverfettung an der selben Tierart nicht ableiten.

Eine Wiederholung der Untersuchungen an einem deutlich erweiterten Datenmaterial sollte unbedingt vorgenommen werden. Wesentliche Nachteile für die Zunahmeleistungen oder den Schlachtkörperwert können bei züchterischer Verbesserung des Futteraufwandes ausgeschlossen werden.

Tabelle 5

Genetische Korrelationen (Standardfehler als Subskript) zwischen der Futteraufnahme (FAP), dem Futteraufwand (FAW) und der täglichen Futteraufnahme (TFM) einerseits und den Merkmalen der Mastleistung und des Schlachtkörperwertes andererseits in Schöndorf und Grub

(Genetic correlations [standard error as subscript] between feed intake [FAP], feed efficiency [FAW] and daily feed intake $[$ TFM] on one hand and fattening performance and carcass quality on the other hand in "Schöndorf" and "Grub")

\begin{tabular}{|c|c|c|c|c|c|c|}
\hline \multirow{2}{*}{ Merkmal } & \multicolumn{3}{|c|}{ Schöndorf (bivariat) } & \multicolumn{3}{|c|}{ Grub (bivariat) } \\
\hline & FAP & FAW & TFM & FAP & FAW & TFM \\
\hline LTZ & $-0,390,17$ & $-0,420,16$ & $-0,490,04$ & $-0,38_{0,11}$ & $-0,41_{0,12}$ & $-0,36_{0,03}$ \\
\hline PTZ & $-0,620,08$ & $-0,690,06$ & $0,58_{0,03}$ & $-0,73_{0,05}$ & $-0,630,06$ & $0,68_{0,03}$ \\
\hline SKK & $-0,43_{0,21}$ & $-0,470,21$ & $-0,070,04$ & $-0,01_{0,13}$ & $0,18_{0,14}$ & $0,05_{0,04}$ \\
\hline SKB & $0,38_{0,26}$ & $0,19_{0,26}$ & $0,26_{0,04}$ & $0,44_{0,17}$ & $0,43_{0,22}$ & $0,17_{0,06}$ \\
\hline SKR & $0,54_{0,23}$ & $0,35_{0,17}$ & $0,320,04$ & $*$ & $*$ & $0,16_{0,09}$ \\
\hline SKF & $0,29_{0,25}$ & $0,41_{0,27}$ & $-0,35_{0,03}$ & $0,37_{0,15}$ & $0,30_{0,16}$ & $-0,12_{0,05}$ \\
\hline NF & $0,12_{0,22}$ & $-0,05_{0,20}$ & $0,31_{0,05}$ & $0,35_{0,10}$ & $0,320,10$ & $-0,001_{0,03}$ \\
\hline USK & $-0,49_{0,21}$ & $-0,40_{0,21}$ & $0,230,04$ & & & \\
\hline USF & $-0,10_{0,29}$ & $-0,020,20$ & $0,59_{0,03}$ & & & \\
\hline KBR & & & & $-0,270,11$ & $-0,12_{0,11}$ & $0,10_{0,03}$ \\
\hline KFL & & & & $0,14_{0,11}$ & $0,14_{0,10}$ & $-0,03_{0,03}$ \\
\hline
\end{tabular}

* $\mathrm{h}^{2}$ Schätzwert $<0,001$

Schlussfolgerungen

Ungünstige korrelierte Selektionseffekte bei Einbeziehung der Futterverwertung/ -aufnahme in die Zuchtwertschätzung auf die Fruchtbarkeit können ausgeschlossen werden. Möglicherweise kann eine effiziente Futterverwertung und eine hohe tägliche Futteraufnahme unter bestimmten Umständen die Fruchtbarkeit verbessern. Allerdings erfordert eine diesbezügliche hohe Aussagesicherheit eine weitere Datensammlung. Auch die hier vorgestellten Untersuchungen bestätigen, dass verbesserte Zunahmeleistungen mit verbessertem Futteraufwand einhergehen.

Hinsichtlich des Schlachtkörperwertes überwiegen die züchterisch wünschenswerten Korrelationen zur Futterverwertung.

Da sich bei der täglichen Futteraufnahme Konflikte hinsichtlich Zunahme und Verfettung andeuten, ist nach derzeitigem Erkenntnisstand die Futteraufwand für die Zuchtarbeit vorzuziehen. 


\section{Literatur}

ARCHER, J.A.; ARTHUR, P.F.; HERD, R.M.; PARNELL, P.F.; PITCHFORD, W.S.:

Optimum postweaning test for measurement of growth rate, feed intake, and feed efficiency in British breed cattle. J. Anim. Sci. 75 (1997), 2024-2032

ARTHUR, P.F.; ARCHER, J.A.; JOHNSTON, D.J.; HERD, R.M.; RICHARDSON, E.C.; PARNELL, P.F.: Genetic and phenotypic variance and covariance components for feed intake, feed efficiency, and other postweaning traits in Angus cattle. J. Anim. Sci. 79 (2001), 2805-2811

BAKER, S.D.; SZASZ, J.I.; KLEIN, T.A.; KUBER, P.S.; HUNT, C.W.; GLAZE, J.B. JR.; FALK, D.; RICHARD, R.; MILLER, J.C.; BATTAGLIA, R.A.; HILL, R.A.:

Residual feed intake of purebred Angus steers: effects on meat quality and palatability. J. Anim. Sci. 84 (2006), 938-945

CAMMACK, K.M.; LEYMASTER, K.A.; JENKINS, T.G.; NIELSEN, M.K.:

Estimates of genetic parameters for feed intake, feeding behavior, and daily gain in composite ram lambs. J Anim Sci. 83 (2005), 777-785

CASTRO BULLE, F.C.; PAULINO, P.V.; SANCHES, A.C.; SAINZ, R.D.:

Growth, carcass quality, and protein and energy metabolism in beef cattle with different growth potentials and residual feed intakes. J. Anim. Sci. 85 (2007), 928-936

FAN, L.Q.; BAILEY, D.R.; SHANNON, N.H.:

Genetic parameter estimation of postweaning gain, feed intake, and feed efficiency for Hereford and Angus bulls fed two different diets. J. Anim. Sci. 73 (1995), 365-72

FRANCOIS, D.; BIBE, D.; BRUNEL, J.B.J.; WEISBECKER, L.; RICARD, E.:

Genetic parameters of feeding traits in meat sheep. Proc. $7^{\text {th }}$ World Congress Gen. Appl. Livest. Prod., Montpellier, France. 2002 CD-ROM Communication 10:10.

GALLIVAN, C.; SULLIVAN, P.G.:

Selection criteria for terminal sires of Ontario lambs. Proc. 5th World. Congr. Genet. Appl. Livest. Prod., Guelph, Canada. 18 (1994), 47-50

GERNAND, E.; LENZ, H.:

Nutzung des Ultraschalls in der Schlachtleistungsprüfung und Zuchtwertschätzung für Schafe. Arch. Tierz. 48 (2005), 174-184

GERNAND, E.; WASSMUTH, R.; LENZ, H.; VON BORSTEL, U.U.; GAULY, M.; KÖNIG, S.:

Impact of energy supply of ewes on genetic parameters for fertility and carcass traits in Merino Long Wool sheep. Small Ruminant Res. (2007) [accepted]

JAKOBSEN, J.H.:

Genetic and phenotypic parameters for traits measured on performance test bulls and the genetic correlation to their daughters yield. M Sc. Speciale 1 Special Husdyravl; L 8332; Institut for Husdyrbrug og Husdyrsundhed; Den. Kgl. Veterinær og Landsbohøjskole København; April 1995

JENSEN, J.; HOHENBROKEN, W.D.; MADSEN, P.; ANDERSON, P.P.

Sire $\times$ nutrition interactions and genetic parameters for energy intake; production an efficiency of nutrient utilization in young bulls; heifers and lactating cows. Acta Agric. Scand. Sect. A; Anim. Sci. 45 (1995), 81-91

KEFELGEN, K.; SÜSS, R.; MIELENZ, N.; SCHÜLER, L.; LENGERKEN VON, G.:

Schätzung genetischer Parameter für Merkmale der Mastleistung und des Schlachtkörperwertes beim Schaf. Arch. Tierz. 41 (1998), 463-472

KENNEDY, B.W.; VAN DER WERF, J.H.; MEUWISSEN, T.H.:

Genetic and statistical properties of residual feed intake. J. Anim. Sci. 71 (1993), 3239-3250

KORMAN, K.:

The effect of the feeding system (ad libitum or rationed) on the feed conversion rate and weight gains of Merino ram lambs. Arch. Tierz. 44 (2001) Special Issue, 240-248

KOVAC, M.; GROENEVELD, E.; GARCIA-CORTEZ, A.: VCE 5.1.2 (2003)

LEYMASTER, K.A.: Straightbred comparison of a composite population and the Suffolk breed for performance traits of sheep. J. Anim. Sci. 69 (1991), 993-999

MAC NEIL, M.D.; BAILEY, D.R.; URICK, J.J.; GILBERT, R.P.; REYNOLDS, W.L.:

Heritabilities and genetic correlations for postweaning growth and feed intake of beef bulls and steers. J. Anim. Sci. 69 (1991), 3183-3189

MADSEN, P.; PETERSEN, G.A.; JENSEN, J.:

Multibred evaluation of bulls performance tested for growth and feed intake based on full and part test period records. Proc. Of the $46^{\text {th }}$ Meeting of the Europeen Association for Animal Production; Prague; 4-7. Sept. 1995 
NIEUWHOF, G.J.; VAN ARENDONK, J.A.M.; VOS, H.; KORVER, S.:

Genetic relationship between feed intake; efficiency and production traits in growing bulls; growing heifers and lactating heifers. Livest. Prod. Sci. 32 (1992), 189-202

NKRUMAH, J.D.; BASARAB, J.A.; PRICE, M.A.; OKINE, E.K.; AMMOURA, A.; GUERCIO, S.; HANSEN, C.; LI, C.; BENKEL, B.; MURDOCH, B.; MOORE, S.S.:

Different measures of energetic efficiency and their phenotypic relationships with growth, feed intake, and ultrasound and carcass merit in hybrid cattle. J Anim Sci. 82 (2004), 2451-2459

NKRUMAH, J.D.; CREWS, D.H. JR.; BASARAB, J.A.; PRICE, M.A.; OKINE, E.K.; WANG, Z.; LI, C.; MOORE: Genetic and phenotypic relationships of feeding behavior and temperament with performance; feed efficiency; ultrasound; and carcass merit of beef cattle. J. Anim. Sci. 85 (2007), 2382-2390

\section{POTTHAST, J.:}

Untersuchungen zur Integration funktionaler Selektionsmerkmale in große Besamungszuchtprogramme bei Milchrindern. Univ. Bonn Diss. (1999)

SNOWDER, G.D.; VAN VLECK, L.D.:

Estimates of genetic parameters and selection strategies to improve the economic efficiency of postweaning growth in lambs. J. Anim. Sci. 81 (2003), 2704-2713

WASSMUTH, R.:

Die Stationäre Futteraufnahmeprüfung von Bullen als Indikator der Gesundheit bei Milchkühen. Habilitationsschrift; Fakultät der Agrarwissenschaften; Univ. Göttingen; Cuvillier Verlag Göttingen (1999)

WASSMUTH, R.; ALPS, H.:

Erfassung der Futteraufnahme im Rahmen der Stationsprüfung potentieller Besamungsbullen. Arch. Tierz. 43 (2000), 561-571

Eingegangen: 2008-04-07

Akzeptiert: 2008-06-17

Autoren:

Dr. ERHARD GERNAND*

E-Mail: e.gernand@clausberg.tll.de

Dr. HEIKE LENZ

E-Mail: h.lenz@clausberg.tll.de

Thüringer Landesanstalt für Landwirtschaft Jena, Abt. Tierproduktion

Standort Clausberg

Clausberg 7

99834 Gerstungen

Deutschland

Prof. Dr. RALF WASSMUTH

E-Mail: ralf.wassmuth@fh-weihenstephan.de

Fachhochschule Weihenstephan, Abt. Triesdorf

Steingruberstraße 2

91746 Weidenbach

Deutschland

Dr. CHRISTIAN MENDEL

E-Mail: christian.mendel@lfl.bayern.de

Bayerische Landesanstalt für Landwirtschaft

Institut für Tierzucht

Prof.-Dürrwaechter Platz 1

85586 Poing-Grub

Deutschland

*korrespondierender Autor 